\title{
Advances in Fusion Gene Research and Fusion Gene Families in Hematological Malignancies
}

\author{
Xue Chen ${ }^{1}$, Fang Wang ${ }^{1}$, Tong Wang ${ }^{1,2}$, Lili Yuan ${ }^{1}$, Hongxing Liu ${ }^{1,2,3^{*}}$ \\ 1Pathology \& Laboratory Medicine Division, Hebei Yanda Lu Daopei Hospital, Langfang, 065201, China \\ ${ }^{2}$ Pathology \& Laboratory Medicine Division, Beijing Lu Daopei Hospital, Beijing, 100176, China \\ ${ }^{3}$ Beijing Lu Daopei Institute of Hematology, Beijing, 100176, China
}

Corresponding Author: Hongxing Liu, MD, Professor, Beijing Lu Daopei Institute of Hematology, 22 South Tongji Road, Beijing, 100076, China. Tel: +86-13693165205, Email: starliu@pku.edu.cn

Received January 13, 2019; Accepted May 8, 2019; Online Published June 15, 2019

\begin{abstract}
Fusion genes (FGs) are major molecular biological abnormalities in hematological malignancies and have become well-established molecular markers for disease classification, risk stratification, and targeted therapies. The long tail phenomenon is observed in the distribution of FGs, which means that except for dozens of the most common FGs, the positive rates of the other FGs are all below $1 \%$, even if they have been frequently reported in the literature. However, the total positive rate of these singly relatively rare FGs is actually not low due to their wide variety and numerous members. We therefore put forward the conception "fusion gene family, FG-FM" to describe fusions that share one common protagonist gene with different partner genes. Most of the FGs in hematological malignancies discovered to date can be classified into about 20 major families. Based on the characteristics of the currently reported FGs, it can be expected that although a great many FGs may be identified in the future, the total number of FG-FMs is rather limited. FGs in the same FG-FM often have similarities in pathogenicity, clinical features, and treatment outcomes. Classifying FGs according to FG-FMs will aid in understanding their pathogenicity and optimizing detection schemes.

Keywords: Gene Fusion, Fusion Gene Family, Long Tail Phenomenon

Citation: Chen X, Wang F, Wang T, Yuan L, Liu H. Advances in fusion gene research and fusion gene families in hematological malignancies. Int J Med Rev. 2019;6(2):45-50. doi:10.29252/IJMR-060204.
\end{abstract}

\section{Introduction}

Chromosomal translocations in hematological malignancies often result in the generation of fusion genes (FGs) and fusion proteins which are usually effective therapeutic targets. ${ }^{1}$ Sometimes the translocations result in abnormally high expression of certain genes without the formation of fusion transcripts or fusion proteins. ${ }^{2,3}$ Once a pathogenic FG exists, it is generally the most important molecular abnormality and has definite clinical implications. A total of 98 FGs were listed in the 2016 revision to the World Health Organization (WHO) classification of tumors of hematopoietic and lymphoid tissues, most of which can serve as molecular markers for the diagnosis and classification of hematological malignancies., ${ }^{4,5}$ Once FGs of pathological significance are detected, they will be deemed as the major molecular etiology of the hematological malignancies and will be monitored by quantitative polymerase chain reaction (qPCR) with high sensitivity. Therefore, FGs can also be used as important molecular markers for evaluating prognosis, selecting targeted therapy or chemotherapy, and monitoring minimal residual disease (MRD). Today, screening multiple FGs simultaneously and then quantitatively monitoring the positive ones has become a routine clinical application..$^{6-8}$

Traditional Methods and Limitations of FGs Identification The traditional FG identification model first identifies the corresponding recurrent chromosomal translocation through karyotype analysis (such as the discovery of the Philadelphia chromosome), and then focuses on the genes which are involved in the chromosomal region and have been broken and misassembled. The most common FGs with characteristic chromosomal translocations in hematological malignancies, such as BCR-ABL1 and PML-RARA, were identified with this strategy. ${ }^{9,10}$ However, it usually takes a long process to identify novel FGs using this method, and it has brought difficulties to applications in ascertaining the FGs caused by rare chromosomal translocations in the past. Moreover, FGs caused by cryptic chromosomal translocations or submicroscopic insertions/deletions without abnormal karyotypes (such as FIP1L1-PDGFRA) are difficult to find and identify with this traditional strategy. ${ }^{11}$

FGs are mutually exclusive, which means that most patients are positive for only one FG. In this study, 36 commonly reported FGs in hematological malignancies in a large cohort

Copyright $\odot 2019$ The Author(s). This is an open-access article distributed under the terms of the Creative Commons Attribution License (http:// creativecommons.org/licenses/by/4.0), which permits unrestricted use, distribution, and reproduction in any medium, provided the original work is properly cited. 
of Chinese acute leukemia patients were retrospectively analyzed. ${ }^{6,7}$ It was found that 23 FGs were detected in 1292 (41.21\%) of 3135 acute myeloid leukemia (AML) cases, and 17 FGs were detected in $712(28.72 \%)$ of 2479 acute lymphoblastic leukemia (ALL) cases. The co-occurrence of two FGs was observed in only $0.22 \%$ of AML and $0.24 \%$ of ALL patients.

The long tail phenomenon is observed in the distribution of FGs. In AML, only 3 FGs (RUNX1-RUNX1T1, PML-RARA and $C B F B-M Y H 11$ ) have a positive rate of more than $5 \%$, and the total positive rate of the other $20 \mathrm{FGs}$ is $13.85 \%$. Similarly, only 2 FGs (BCR-ABL1 and ETV6-RUNX1) in ALL have a positive rate of more than $5 \%$, and the total positive rate of the other 15 FGs is $10.93 \%$. Except for the most common 13 FGs, the individual positive rates of the other FGs are all below 1\% even if they have been frequently reported in the literature. The long tail distribution phenomenon of FGs has also been supported by several other systematic research reports. ${ }^{12-14}$

Another feature of the long tail phenomenon is that the total positive rate of all singly relatively rare FGs is actually not low due to their wide variety and numerous members. ${ }^{13,14}$ To date, the exact population of FGs undiscovered in hematological malignancies and the total positive rate of them remain unknown. For most FGs with pathological significance, even if the positive rate of one single FG is relatively low, it still has definite significance in clinical diagnosis, treatment guidance, and MRD monitoring. ${ }^{15,16}$ Thus, the detection of these rare FGs is also clinically significant. For example, FIP1L1PDGFRA is rare in hematological malignancies; however, the FIP1L1-PDGFRA fusion protein is 100 times more sensitive than $B C R-A B L 1$ to imatinib. ${ }^{4,15}$ Even in the routine detection of FGs that are already well known, false negative results may occur because of variant fusion transcripts, such as $B C R-A B L 1$ and their variant and rare isotypes. ${ }^{17,18}$ Therefore, how to effectively identify rare and variant FGs poses great challenges for detection methods and data analysis.

\section{Technological Advances and New Discoveries}

The rapid development of sequencing technology and the decline of sequencing costs in recent years have made next generation sequencing (NGS), including whole genome sequencing (WGS), whole exome sequencing (WES), and whole transcriptome sequencing (WTS), more accessible. ${ }^{13,14,16}$ WGS can identify breakpoints and splicing sites at the genomic level, while WTS can directly identify abnormal transcripts. WGS and WTS can not only analyze known FGs, but also have unique advantages for identification of unknown rare and variant FGs. ${ }^{19,20}$ In a study of 231 pediatric patients with ALL using WTS, ${ }^{21} 58$ putative functional and predominant FGs were identified in $54.1 \%$ of patients, 31 of which were newly discovered. Thanks to the promising application of NGS, a large number of newly identified FGs have been reported in recent years, and new classification patterns of hematological malignancies have been suggested. ${ }^{13,14,21,22}$

$B C R-A B L 1$-like ALL, which is now incorporated into the 2016 revision of the WHO classification of neoplastic diseases of the hematopoietic and lymphoid tissues as a provisional entity, is a subgroup of ALL characterized by a gene-expression profile similar to that of $B C R-A B L 1$ positive ALL, alterations of lymphoid transcription factor genes, and a poor outcome. ${ }^{5,22,23} \mathrm{~A}$ wide range of genetic alterations that dysregulate several cytokine receptors and kinase signaling pathways (predominantly ABL and Janus kinases class), including gene rearrangements involving genes including $A B L 1, A B L 2, C R L F 2, C S F 1 R, E P O R$, JAK2, NTRK3, and $P D G F R B$, are found in $B C R-A B L 1$-like ALL. ${ }^{24,25}$ It is a common subtype of B-ALL, representing 7\%-25\% of new diagnoses, but the incidence of a specific FG is rather rare. ${ }^{26-30}$ So far, hundreds of kinase-related FGs in BCR-ABL1-like ALL have been reported, yet most of them are only seen in single cases in reports. Therefore, it is difficult to study and classify this subgroup of diseases based on the conventional strategy of single gene abnormality identification. Considering that $B C R-A B L 1$-like ALL could benefit from tyrosine kinase inhibitor (TKI) therapy, the application of integrated genomic analysis to improve diagnoses is of vital clinical significance.

$\mathrm{Gu}$ et al recently reported their systematic study on a large cohort of 1988 B-ALL subjects to identify chromosomal rearrangements, gene expression profiles, and large-scale copy number alterations. ${ }^{14}$ They found that totally $7.4 \%$ patients have PAX5 alterations including rearrangements, intragenic amplifications, or non-PAX5 P80R mutations. Children in this subtype were more commonly classified as high risk rather than standard risk. $38.5 \%$ of them harbored $P A X 5$ rearrangements involving 24 partner genes, the most frequent of which were PAX5-ETV6, PAX5-NOL4L, PAX5AUTS2, and PAX5-CBFA2T3. The long tail phenomenon was also observed in the distribution of PAX5-FGs. ${ }^{14}$

The ZNF384 gene is located close to the telomeric end of the short arm of chromosome 12, making identification of translocations involving ZNF384 challenging by G-band analysis. WTS found that the total frequency of ZNF384-FGs was $4.1 \%$ in $291 \mathrm{~B}$-ALL patients, and TCF3-ZNF384 was the most recurrent with a frequency of $2.4 \% .{ }^{31}$ Patients harboring ZNF384-FGs have a common immunophenotype of weak CD10 and abnormal CD13 and/or CD33 expression. The gene expression signature of TCF3-ZNF384-positive patients was similar to that of patients with other ZNF384-FGs, but it was significantly different from that of TCF3-PBX1positive and ZNF384-FGs-negative ALL patients. However, the clinical features of TCF3-ZNF384-positive patients were markedly different from those of EP300-ZNF384-positive patients, exhibiting higher cell counts and a younger age at presentation. Patients harboring TCF3-ZNF384 showed a poor response to steroid and a high frequency of relapse, and some of them had additional activating mutations in RAS signaling pathway genes. ${ }^{31}$ Another research showed that EP300-ZNF384 and CREBBP-ZNF384 fusions resulted in loss of histone lysine acetyltransferase activity and increased sensitivity of leukemia cells to histone deacetylase inhibitors. EP300-ZNF384 and CREBBP-ZNF384 may cause epigenetic deregulation with the potential for therapeutic targeting. ${ }^{21}$ These studies indicate that ZNF384-FGs-positive ALL has characteristic immunophenotype and can be clustered into 
one subtype of ALL. On the other hand, the clinical features, therapy, and prognosis may be influenced by the functional properties of individual fusion partners. ${ }^{21,32}$

FGs involving $M E F 2 D$ have recently been identified in B-ALL, and the incidence of $M E F 2 D$-FGs overall was $2.4 \%$ in consecutive B-ALL patients in a single clinical trial. ${ }^{33,34} \mathrm{MEF} 2 \mathrm{D}$ FGs-positive patients frequently presented a cytoplasmic $\mu$ chain-positive, pre-B immunophenotype and often expressed an aberrant CD5 antigen. Elevated GATA3 expression was also a characteristic feature of MEF2D-FGs-positive patients, and PHF6 mutations showed an unexpectedly high frequency (50\%) in them. MEF2D-FGs-positive patients were older (median age 9 years) with elevated WBC counts at the time of diagnosis and were mostly classified as NCI (National Cancer Institute) high risk. Although they responded well to steroid treatment, MEF2D-FGs-positive patients showed a significantly worse outcome with more than half of them suffering relapse and death. Relapse was frequently associated with CDKN2A/CDKN2B deletions. Therefore, MEF2D-FGs represent a distinct subgroup of B-ALL with characteristic immunophenotype and gene expression signatures and poor prognoses. $^{33-35}$

\section{Classification and Recognition of FGs as "Fusion Gene Family"}

For a better understanding of the incidences of FGs and their pathological characteristics, we put forward the conception of "fusion gene family, FG-FM" to describe fusions which involve one protagonist gene and multiple fusion partners (Figure 1). Hundreds of FGs have been reported in hematological malignancies so far, and novel
FGs may be reported at any time; most of them are rare in individuals. ${ }^{4,519}$ The study of their functions and pathogenic mechanisms as well as the choice of detection methods are often confusing. On the other hand, family aggregation phenomenon is quite common, and FGs in the same family often have commonalities in pathogenicity, clinical features, and treatment outcomes. ${ }^{28-30}$ Therefore, classifying FGs according to FG-FMs will aid in better understanding their pathological significance and suggesting new classification patterns of hematological malignancies.

KMT2A-FM is a large FG-FM which has been systematically studied and reported in hematologicalmalignancies. ${ }^{36} \mathrm{KMT} 2 \mathrm{~A}$ FM has lots of members and some of them are more likely to occur in certain types of leukemia. For instance, KMT2AELL is found almost exclusively in AML, KMT2A-AFF1 is more likely to occur in ALL, while KMT2A-MLLT3 is prone to occur in AML and infant ALL., ${ }^{4,5} \mathrm{New}$ and rare KMT2AFGs are constantly being reported..$^{20}$ Meyer et al. identified 11 novel partner genes of KMT2A in 2345 acute leukemia cases with $K M T 2 A$ rearrangement.$^{36}$ They summarized that a total of 135 different KMT2A rearrangements have been identified so far, of which 94 KMT2A-FGs have been characterized at the molecular level. Thirty-five of the 94 KMT2A-FGs occur recurrently, while 9 of them account for more than $90 \%$ of all FGs. The distribution of KMT2A-FM members also reveals a prominent long tail characteristic.

Because KMT2A has a large amount of known and an even much greater number of unknown partners, it is difficult to accurately calculate the positive rate of KMT2A-FGs. It is not uncommon to find chromosome translocations involving 11q23, where the KMT2A gene is located, but without

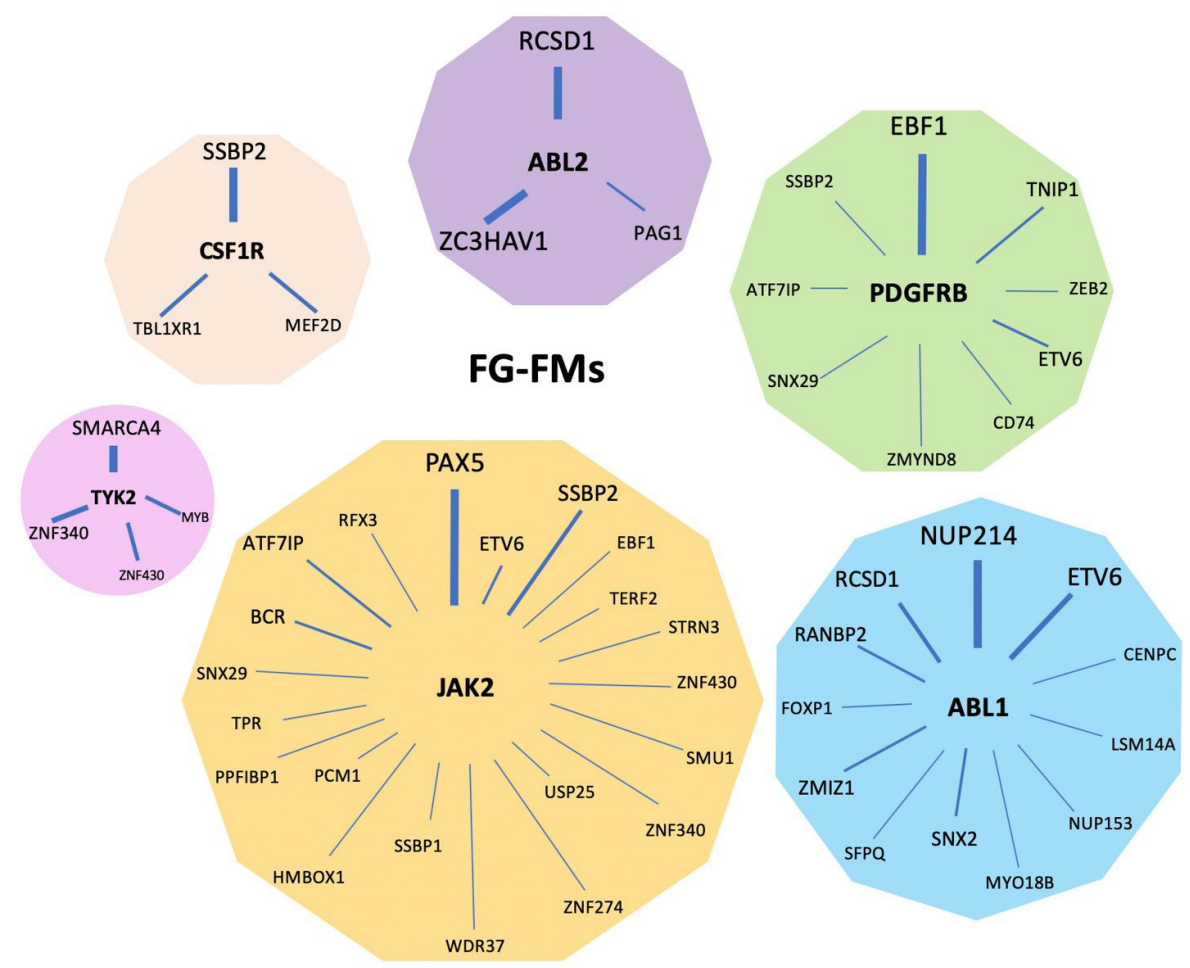

Figure 1. Summary View of the Key Fusion Families (FG-FMs) in BCR-ABL1-like ALL. Each colored region represents an FG-FM. The lines connecting gene symbols indicate fusion partners. The thickness of each line reflects the frequency of the observed fusion. 
detectable relatively common KMT2A-FGs due to the long tail effect. The translocations may result in rare known or unknown KMT2A-FGs or occasionally involve other genes (e.g., ZBTB16) rather than KMT2A in this chromosome region. ${ }^{37}$

Another FG-FM with numerous family members in hematological malignancies is PDGFRB-FM. About 30 PDGFRB-FGs have been recorded in the 2016 revision to the WHO classification of tumors of hematopoietic and lymphoid tissues, with TEL-PDGFRB being the most common one. Most of them are sensitive to TKIs such as imatinib. ${ }^{4}$ Some PDGFRB-FGs are seen in BCR-ABL1-like B-ALL and are also sensitive to TKIs. ${ }^{4,16}$ However, most of the PDGFRB-FGs are reported only in single cases, thus exerting great difficulties in detection and applying targeted therapy.

According to the concept of "FG-FM" and recent literature, the 98 FGs listed in the 2016 revision to the WHO classification of tumors of hematopoietic and lymphoid tissues can be classified into 18 FG-FMs, such as KMT2A-FM, RARA-FM, PDGFRA-FM, and PDGFRB-FM, with only 12 FGs like CBFB-MYH11. STIL-TAL1 cannot be classified into any family. ${ }^{4.5}$ Along with the MEF2D-FM, ZNF384-FM, and PAX5-FM, which were recently reported in the literature, ${ }^{14,31-35}$ most of the FGs in hematological malignancies discovered to date can be clustered into about 20 major FG-FMs (Table 1). Furthermore, the content of FGs that can be covered in these FG-FMs far exceeds the known FGs in de facto. Based on

Table 1. Gene Translocations Reported in WHO 2016 and Recent Literature

\begin{tabular}{lcc}
\hline FG-FMs & $\begin{array}{c}\text { FGs not Belonging to } \\
\text { any FG-FMs }\end{array}$ & $\begin{array}{c}\text { Gene Translocations Without } \\
\text { Fusion Proteins }\end{array}$ \\
\hline ABL1-FM & BIRC3-MALT1 & BCL2-TL \\
ABL2-FM & CBFA2T3-GLIS2 & BCL6-TL \\
ALK-FM & CBFB-MYH11 & CD274 (PDL1)-TL \\
CSF1R-FM & CTLA-CD28 & CIITA-TL \\
ETV6-FM & DEK-NUP214 & CRLF2-TL \\
FGFR1-FM & ITK-SYK & DUSP22-TL \\
IAK2-FM & KAT6A-CREBBP & DUX4-TL* \\
KMT2A-FM & MYB-GATA1 & EPOR-TL \\
MEF2D-FM* & NPM1-TYK2 & IGH@-TL \\
NTRK3-FM & PICALM-MLLT10 & MYC-TL \\
NUP98-FM & RBM15-MKL1 & PDCD1LG2 (PDL2)-TL \\
PAX5-FM* & STIL-TAL1 & TCR@-TL \\
PDGFRA-FM & & \\
PDGFRB-FM & & \\
RARA-FM & & \\
RUNX1-FM & & \\
TBL1XR1-FM & & \\
TCF3-FM & & \\
TP63-FM & & \\
TYK2-FM & & \\
ZNF384-FM* & & \\
\hline FbF & & \\
\hline
\end{tabular}

Abbreviations: FG, fusion gene; FM, family; TL, translocation.

* indicates the FG-FM or gene-TL not mentioned in the WHO 2016, but reported in recent literature. the characteristics of the currently reported FGs, it can be expected that although numerous FGs may be identified in the future, the total number of FG-FMs is rather limited.

\section{Challenges in the Detection and Application of FGs}

Genomic analysis has now provided generic methods for FG identification; however, high experimental costs and analytical thresholds are still being faced. Moreover, WGS or WTS generate a lot of background sequences and many abnormal splicing sequences without pathological significance. ${ }^{21}$ How to effectively analyze and differentiate the key FG sequences from multitudinous background sequences is a great challenge to the analyst's professional background and analytical capabilities. Care should also be taken to avoid false negative results caused by methodologies or analysis procedures.

FGs in the same FG-FM have commonalities in pathological and clinical features in general, and the concept of FG-FM will help to speculate on and study the functions of newly identified FGs. Notably, attention should also be paid to the influence of partner genes on the pathogenicity of FGs. If both partners of one FG are protagonist genes of one FG-FM, the FG should be classified mainly according to its major pathogenicity and corresponding clinical features. For example, the gene expression signature and clinical manifestations of PAX5$J A K 2$-positive ALL are similar to that of $B C R$ - $A B L 1$-like ALL, so PAX5-JAK2 should be classified into the JAK2-FM rather than the PAX5-FM. ${ }^{14,22}$

The majority of FGs with pathogenicity can be used as molecular markers for MRD monitoring after treatment. As the most common FG in hematological malignancies, the quantitative standardization of $B C R-A B L 1$ has been carried out for more than ten years, but there are still many problems to be solved. ${ }^{38,39}$ Every FG-FM may consist of numerous but individually fairly rare FGs. How to effectively design suitable detection methods and how to promote the standardization of quantitative testing need further optimized solutions. For the quantitative detection of rare FGs, using internationally traceable standardized samples is not practical. Digital PCR technology can provide absolute quantification without calibrator and standard curve, yet a specific detection method still needs to be designed for each FG. Digital PCR technology is limited to transcript type, such as $B C R-A B L 1$ ela2 and e13a2..$^{40}$

\section{Conclusions}

Massive genomic sequencing and analysis can aid the identification of FGs, especially the rare ones, and the further exploration of their molecular etiology in hematological malignancies. The concept of FG-FM simplifies the understanding of FGs based on their functional clustering and pathogenicity. At the same time, it also brings some new challenges to designing suitable detection methods, which needs persistent efforts together with technological progression and an understanding of the panoramic view of FGs and FG-FMs in hematological malignancies. 


\section{Authors' Contributions}

All authors contributed equally to this study.

\section{Conflict of Interest Disclosures}

The authors have no conflicts of interest to disclose.

\section{Acknowledgments}

There are no particular acknowledgments to declare for this review article.

\section{References}

1. Montano A, Forero-Castro M, Marchena-Mendoza D, Benito R, Hernandez-Rivas JM. New challenges in targeting signaling pathways in acute lymphoblastic leukemia by NGS approaches: An update. Cancers (Basel). 2018;10(4). doi:10.3390/ cancers10040110.

2. Chisholm KM, Mohlman J, Liew M, et al. IRF4 translocation status in pediatric follicular and diffuse large B-cell lymphoma patients enrolled in Children's Oncology Group trials. Pediatr Blood Cancer. 2019:e27770. doi:10.1002/pbc.27770.

3. Martin-Garcia D, Navarro A, Valdes-Mas R, et al. CCND2 and CCND3 hijack immunoglobulin light-chain enhancers in cyclin D1(-) mantle cell lymphoma. Blood. 2019;133(9):940-951. doi:10.1182/blood-2018-07-862151.

4. Arber DA, Orazi A, Hasserjian R, et al. The 2016 revision to the World Health Organization classification of myeloid neoplasms and acute leukemia. Blood. 2016;127(20):2391-2405. doi:10.1182/blood-2016-03-643544.

5. Swerdlow SH, Campo E, Pileri SA, et al. The 2016 revision of the World Health Organization classification of lymphoid neoplasms. Blood. 2016;127(20):2375-2390. doi:10.1182/ blood-2016-01-643569.

6. Chen X, Wang F, Zhang Y, et al. Retrospective analysis of 36 fusion genes in 2479 Chinese patients of de novo acute lymphoblastic leukemia. Leuk Res. 2018;72:99-104. doi:10.1016/j. leukres.2018.08.009.

7. Chen X, Wang F, Zhang Y, et al. Panoramic view of common fusion genes in a large cohort of Chinese de novo acute myeloid leukemia patients. Leuk Lymphoma. 2019;60(4):1071-1078. doi:1 0.1080/10428194.2018.1516876.

8. Chen X, Wang F, Fang J, et al. Analysis of 36 fusion genes in 595 cases of de novo pediatric acute myeloid leukemia. J Clin Pathol Res. 2018,38(6):1202-1207. doi:10.3978/j.issn.20956959.2018.06.011.

9. Mughal TI, Radich JP, Deininger MW, et al. Chronic myeloid leukemia: reminiscences and dreams. Haematologica. 2016;101(5):541-558. doi:10.3324/haematol.2015.139337.

10. Chen SJ, Zhou GB, Zhang XW, Mao JH, de The H, Chen Z. From an old remedy to a magic bullet: molecular mechanisms underlying the therapeutic effects of arsenic in fighting leukemia. Blood. 2011;117(24):6425-6437. doi:10.1182/blood-2010-11-283598.

11. Kim TH, Gu HJ, Lee WI, Lee J, Yoon HJ, Park TS. Chronic eosinophilic leukemia with FIP1L1-PDGFRA rearrangement. Blood Res. 2016;51(3):204-206. doi:10.5045/br.2016.51.3.204.

12. Zaliova M, Stuchly J, Winkowska L, et al. Genomic landscape of pediatric B-other acute lymphoblastic leukemia in a consecutive European cohort. Haematologica. 2019. doi:10.3324/ haematol.2018.204974.

13. Li JF, Dai YT, Lilljebjorn $H$, et al. Transcriptional landscape of B cell precursor acute lymphoblastic leukemia based on an international study of 1,223 cases. Proc Natl Acad Sci U S A. 2018;115(50):E11711-e11720. doi:10.1073/pnas.1814397115.

14. Gu Z, Churchman ML, Roberts KG, et al. PAX5-driven subtypes of B-progenitor acute lymphoblastic leukemia. Nat Genet.

\section{9;51(2):296-307. doi:10.1038/s41588-018-0315-5.}

15. Srinivasan A, Scordino T, Baker A. Myeloid Neoplasm With Eosinophilia and FIP1L1-PDGFRA Rearrangement Treated With Imatinib Mesylate: A Pediatric Case With Long-term Follow-up. J Pediatr Hematol Oncol. 2019;41(4):334-335. doi:10.1097/ mph.0000000000001446.

16. Such E, Liquori A, Mora E, et al. RNA Sequencing Analysis for the Identification of a PCM1/PDGFRB Fusion Gene Responsive to Imatinib. Acta Haematol. 2019:1-6. doi:10.1159/000497348.

17. Liu HX, Zhu P. Comprehensive Testing Strategy of BCR-ABL1 Fusion Gene and Clinical Guidance. Chinese Journal of Clinicians (Electronic Edition). 2008;2:826-831. doi:10.3969/j.issn.16740785.2008.07.017

18. Piedimonte $M$, Ottone $T$, Alfonso $V$, et al. A rare BCR-ABL1 transcript in Philadelphia-positive acute myeloid leukemia: case report and literature review. BMC Cancer. 2019;19(1):50. doi:10.1186/s12885-019-5265-5.

19. Chen $X$, Wang $F$, Zhang $Y$, et al. A novel NPM1-RARG-NPM1 chimeric fusion in acute myeloid leukaemia resembling acute promyelocytic leukaemia but resistant to all-trans retinoic acid and arsenic trioxide. Br J Cancer. 2019;120(11):1023-1025. doi:10.1038/s41416-019-0456-z.

20. Ikeda J, Shiba N, Tsujimoto SI, et al. Whole transcriptome sequencing reveals a KMT2A-USP2 fusion in infant acute myeloid leukemia. Genes Chromosomes Cancer. 2019. doi:10.1002/ gcc. 22751 .

21. Qian M, Zhang $H$, Kham SK, et al. Whole-transcriptome sequencing identifies a distinct subtype of acute lymphoblastic leukemia with predominant genomic abnormalities of EP300 and CREBBP. Genome Res. 2017;27(2):185-195. doi:10.1101/ gr.209163.116.

22. Zhang Y, Liu HX. Progression of molecular genetics in Ph-like acute lymphoblastic leukemia: Reports from the 56th American society of hematology annual meeting. J Leuk Lymphoma. 2015;24(2):7478. doi:10.3760/cma.j.issn.1009-9921.2015.02.003.

23. Roberts KG, LiY, Payne-Turner D, et al. Targetable kinase-activating lesions in Ph-like acute lymphoblastic leukemia. N Engl J Med. 2014;371(11):1005-1015. doi:10.1056/NEJMoa1403088.

24. Pui $\mathrm{CH}$, Roberts KG, Yang JJ, Mullighan CG. Philadelphia chromosome-like acute lymphoblastic leukemia. Clin Lymphoma Myeloma Leuk. 2017;17(8):464-470. doi:10.1016/j. clml.2017.03.299.

25. Siegele BJ, Nardi V. Laboratory testing in BCR-ABL1-like (Philadelphia-like) B-lymphoblastic leukemia/lymphoma. Am J Hematol. 2018;93(7):971-977. doi:10.1002/ajh.25126.

26. van der Veer A, Waanders E, Pieters R, et al. Independent prognostic value of BCR-ABL1-like signature and IKZF1 deletion, but not high CRLF2 expression, in children with B-cell precursor ALL. Blood. 2013;122(15):2622-2629. doi:10.1182/blood-2012-10-462358.

27. Tasian SK, Hurtz C, Wertheim GB, et al. High incidence of Philadelphia chromosome-like acute lymphoblastic leukemia in older adults with B-ALL. Leukemia. 2017;31(4):981-984. doi:10.1038/leu.2016.375.

28. Herold T, Baldus CD, Gokbuget N. Ph-like acute lymphoblastic leukemia in older adults. N Engl J Med. 2014;371(23):2235. doi:10.1056/NEJMc1412123.

29. Boer JM, Koenders JE, van der Holt B, et al. Expression profiling of adult acute lymphoblastic leukemia identifies a BCR-ABL1like subgroup characterized by high non-response and relapse rates. Haematologica. 2015;100(7):e261-264. doi:10.3324/ haematol.2014.117424.

30. Tasian SK, Loh ML, Hunger SP. Philadelphia chromosome-like acute lymphoblastic leukemia. Blood. 2017;130(19):2064-2072. doi:10.1182/blood-2017-06-743252.

31. Hirabayashi S, Ohki K, Nakabayashi K, et al. ZNF384-related 
fusion genes define a subgroup of childhood B-cell precursor acute lymphoblastic leukemia with a characteristic immunotype. Haematologica. 2017;102(1):118-129. doi:10.3324/ haematol.2016.151035.

32. McClure BJ, Heatley SL, Kok CH, et al. Pre-B acute lymphoblastic leukaemia recurrent fusion, EP300-ZNF384, is associated with a distinct gene expression. Br J Cancer. 2018;118(7):1000-1004. doi:10.1038/s41416-018-0022-0.

33. Ohki K, Kiyokawa N, Saito $Y$, et al. Clinical and molecular characteristics of MEF2D fusion-positive B-cell precursor acute lymphoblastic leukemia in childhood, including a novel translocation resulting in MEF2D-HNRNPH1 gene fusion. Haematologica. 2019;104(1):128-137. doi:10.3324/ haematol.2017.186320.

34. Gu Z, Churchman M, Roberts K, et al. Genomic analyses identify recurrent MEF2D fusions in acute lymphoblastic leukaemia. Nat Commun. 2016;7:13331. doi:10.1038/ncomms13331.

35. Lilljebjorn $\mathrm{H}$, Fioretos $\mathrm{T}$. New oncogenic subtypes in pediatric B-cell precursor acute lymphoblastic leukemia. Blood. 2017;130(12):1395-1401. doi:10.1182/blood-2017-05-742643.
36. Meyer C, Burmeister $\mathrm{T}$, Groger $\mathrm{D}$, et al. The MLL recombinome of acute leukemias in 2017. Leukemia. 2018;32(2):273-284. doi:10.1038/leu.2017.213.

37. Lechevalier N, Dulucq S, Bidet A. A case of acute promyelocytic leukaemia with unusual cytological features and a ZBTB16RARA fusion gene. Br J Haematol. 2016;174(4):502. doi:10.1111/ bjh.14198.

38. Cross NC, White HE, Ernst T, et al. Development and evaluation of a secondary reference panel for BCR-ABL1 quantification on the International Scale. Leukemia. 2016;30(9):1844-1852. doi:10.1038/leu.2016.90.

39. Pfeifer H, Cazzaniga G, van der Velden VHJ, et al. Standardisation and consensus guidelines for minimal residual disease assessment in Philadelphia-positive acute lymphoblastic leukemia (Ph + ALL) by real-time quantitative reverse transcriptase PCR of e1a2 BCRABL1. Leukemia. 2019. doi:10.1038/s41375-019-0413-0.

40. Dogliotti I, Drandi D, Genuardi E, Ferrero S. New molecular technologies for minimal residual disease evaluation in B-Cell lymphoid malignancies. J Clin Med. 2018;7(9). doi:10.3390/ jcm7090288. 